\title{
COPELAND'S DEFINITION OF A STIELTJES INTEGRAL
}

\author{
R. L. JEFFERY
}

Our present interest in Copeland's definition ${ }^{1}$ of the Stieltjes integral of $g(x)$ with respect to the monotone function $f(x)$ is due to a remark by T. H. Hildebrandt ${ }^{2}$ to the effect that in the proof of the formula for integration by parts it is required that $f(x)=\frac{1}{2}\{f(x+0)+f(x-0)\}$. In looking over Copeland's paper it was found that it is only in the proof of this formula that $f(x)$ is so restricted. Furthermore, it became clear that the definition possesses a considerable degree of generality. In the present note we simplify the definition, and compare it with that of the Riemann-Stieltjes integral and the Lebesgue-Stieltjes integral.

The classical definition of a Riemann-Stieltjes integral is

$$
\operatorname{RS} \int_{\alpha}^{\beta} g d f=\lim _{n \rightarrow \infty} \sum_{k=1}^{n} g\left(\xi_{k}\right)\left\{f\left(x_{k}\right)-f\left(x_{k-1}\right)\right\},
$$

where $\left(x_{k-1}, x_{k}\right)$ is a finite subdivision of the interval $\alpha \leqq x \leqq \beta$, with $x_{i}-x_{i-1} \rightarrow 0$ and $\xi_{k}$ any point on $\left(x_{k-1}, x_{k}\right)$. The limit (1) exists when $g$ is continuous, but may fail to exist even for functions $g$ of bounded variation unless further restrictions ${ }^{3}$ are placed on the subdivision $\left(x_{k-1}, x_{k}\right)$ or on the choice of $\xi_{k}$. Copeland's definition is likewise based on what can be interpreted as a sequence of finite sets $\left\{\left(x_{k}\right)\right\}$, $k=1,2, \cdots, n$, of $(\alpha, \beta)$, and the integral is given by

$$
\operatorname{CS} \int_{\alpha}^{\beta} g d f=\lim _{n \rightarrow \infty} \frac{g\left(x_{1}\right)+\cdots+g\left(x_{n}\right)}{n} .
$$

The sequence $\left\{x_{k}\right\}$ is defined wholly in terms of $f$, and the limit (2) exists for a wide class of functions including functions of bounded variation.

The set $\left\{x_{k}\right\}, k=1,2, \cdots, n ; n=1,2, \cdots$, on which (2) is based is denumerable. Consequently the value of the integral depends only on the values of $g$ over this denumerable set, which permits $g$ an undesirable amount of freedom. To obviate this defect, and to bring the definition more in line with that of the Riemann-Stieltjes integral, we introduce some changes in its formulation.

On the interval $\alpha \leqq x \leqq \beta$ let $g(x)$ be bounded, and $f(x)$ be bounded

${ }_{1}^{1}$ This Bulletin, vol. 43 (1937), pp. 581-588.

2 American Mathematical Monthly, vol. 45 (1938), p. 277.

${ }^{3}$ This point has been thoroughly covered by Hildebrandt, loc. cit., $\S \S 6,7,8,9$. 
and non-decreasing. In order to keep the initial stages as simple as possible, we shall assume that $f(\alpha+0)=0, f(\beta-0)=1$. This restriction will be removed later. For a given $n$ and for $k=1,2, \cdots, n-1$ let $x_{k}$ be the greatest lower bound of numbers $x$ for which

$$
f(x-0) \leqq k / n \leqq f(x+0) \text {. }
$$

The numbers $x_{k},(k=1,2, \cdots, n-1)$, all lie on the open interval $\alpha<x<\beta$. Let $a<x<b$ be an open interval on $\alpha<x<\beta$, and for any $n$ let $p$ be the number of points of the set $\left\{x_{k}\right\}$ on $a<x<b$. Then

$$
f(b-0)-f(a+0)-1 / n<p / n<f(b-0)-f(a+0)+1 / n,
$$

from which it follows that

$$
\lim _{n \rightarrow \infty} p / n=f(b-0)-f(a+0)
$$

Also if $x_{0}$ is a point of discontinuity of $f$ on $\alpha<x<\beta$ and $q$ is the number of times $x_{0}$ is repeated in the set $\left\{x_{k}\right\}$, then

$$
f\left(x_{0}-0\right)-1 / n<q / n<f\left(x_{0}+0\right)+1 / n,
$$

which gives

$$
\lim _{n \rightarrow \infty} q / n=f\left(x_{0}+0\right)-f\left(x_{0}-0\right) \text {. }
$$

The set $\left\{x_{k}\right\}, k=1,2, \cdots, n-1$, is non-decreasing on $\alpha<x<\beta$. Let $\xi_{1}$ be a point on $\alpha<x<x_{1}, \xi_{k}$ a point on $x_{k-1} \leqq x \leqq x_{k}$ if $x_{k} \neq x_{k-1}, \xi_{k}=x_{k}$ if $x_{k}=x_{k-1}, k=2,3, \cdots, n-1$, and $\xi_{n}$ a point on $x_{n-1}<x<\beta$. Form the sum

$$
G_{n}=\frac{g\left(\xi_{1}\right)+\cdots+g\left(\xi_{n}\right)}{n}
$$

and over the open interval $\alpha<x<\beta$ define

$$
\operatorname{CS} \int_{\alpha<x<\beta} g d f=\lim _{n \rightarrow \infty} G_{n}
$$

provided this limit exists. If $x_{0}$ is a point of discontinuity of $f$ on $\alpha<x<\beta$, it follows from (5) that the part of $G_{n}$ arising from $x_{0}$ tends to $g\left(x_{0}\right)\left\{f\left(x_{0}+0\right)-f\left(x_{0}-0\right)\right\}$. This leads us to define

$$
\operatorname{CS} \int_{x_{0}} g d f=g\left(x_{0}\right)\left\{f\left(x_{0}+0\right)-f\left(x_{0}-0\right)\right\},
$$

which may be retained when $x_{0}$ is a point of continuity of $f$. If $a<x<b$ 
is an open interval on $\alpha<x<\beta$, set $g^{\prime}=g$ on $a<x<b, g^{\prime}=0$ elsewhere on $\alpha<x<\beta$, and define

$$
\operatorname{CS} \int_{a<x<b} g d f=\operatorname{CS} \int_{\alpha<x<\beta} g^{\prime} d f .
$$

We further define

$$
\begin{aligned}
\operatorname{CS} \int_{a}^{b} g d f= & \operatorname{CS} \int_{a<x<b} g d f+g(a)\{f(a+0)-f(a)\} \\
& +g(b)\{f(b)-f(b-0)\} .
\end{aligned}
$$

From (7) and (8) we see that

$$
\operatorname{CS} \int_{a}^{b} g d f=\int_{a<x<b} g d f+\int_{a} g d f+\int_{b} g d f
$$

only if $f(a)=f(a-0)$, and $f(b)=f(b+0)$. It is true, however, that if $c$ is a point on $a<x<b$, then

$$
\operatorname{CS} \int_{a}^{b} g d f=\operatorname{CS} \int_{a}^{c} g d f+\operatorname{CS} \int_{c}^{b} g d f .
$$

With the restrictions $f(\alpha+0)=0, f(\beta-0)=1$ still holding, the Copeland-Stieltjes integral, $\operatorname{CS} \int_{a}^{b} g d f$, has now been defined for every interval $(a, b)$ on $\alpha \leqq x \leqq \beta$. We shall prove the following theorems.

THEOREM I. The necessary and sufficient condition for the existence of the CS-integral is that the common part of the discontinuities of $g$ and the continuities of $f$ have zero measure with respect to $f$.

Theorem II. If the CS-integral exists, the LS-integral (LebesgueStieltjes integral) exists, and the two are equal.

THEOREM III. If $F(x)=\int_{\alpha}^{x} g d f$, then $d F / d f$ exists and is equal to $g$ except for at most a set of zero measure with respect to $f$.

It is thus seen that CS-integration with respect to the monotone function $f(x)$ has the same degree of generality as ordinary Riemann integration with respect to the variable $x$. In other words: If Copeland's definition replaces that of Riemann, it makes no difference whether the variable of integration is $x$ or a monotone function $f(x)$.

Let $D=d_{1}, d_{2}, \cdots$ be the points of discontinuity of $f$, and for any point $d_{i}$ let $\left(a_{i}, a_{i}^{\prime}\right)$ be an interval with $a_{i}<d_{i}<a_{i}^{\prime}$ and for which $a_{i}$ and $a_{i}^{\prime}$ are points of continuity of $f$. Let $p_{i}, q_{i}, r_{i}$ be respectively the number of points of the set $\left\{x_{k}\right\}$ on $a_{i}<x_{i}<d_{i}$, the number of times $d_{i}$ 
is repeated in this set, and the number of points of the set on $d_{i}<x<a_{i}^{\prime}$. Then $p_{i} / n \rightarrow f\left(d_{i}-0\right)-f\left(a_{i}\right), q_{i} / n \rightarrow f\left(d_{i}+0\right)-f\left(d_{i}-0\right)$, and $r_{i} / n \rightarrow f\left(a_{i}^{\prime}\right)-f\left(d_{i}+0\right)$. Thus if $a_{i}^{\prime}-a_{i}$ is sufficiently small and $n$ is sufficiently great, the part of $G_{n}$ arising from the interval $\left(a_{i}, a_{i}^{\prime}\right)$ is arbitrarily near $g\left(d_{i}\right)\left\{f\left(d_{i}+0\right)-f\left(d_{i}-0\right)\right\}=\operatorname{CS} \int_{d_{i}} g d f$. Since $g$ is bounded and $f$ is bounded and non-decreasing, $\sum g\left(d_{i}\right)\left\{f\left(d_{i}+0\right)-f\left(d_{i}-0\right)\right\}$ converges. Also, there exists a set of nonoverlapping intervals $A=\left(a_{1}, a_{1}^{\prime}\right), \cdots,\left(a_{l}, a_{l}^{\prime}\right)$ with $a_{i}<d_{i}<a_{i}^{\prime}$ and such that for $l$ sufficiently great and $n$ sufficiently great we have

$$
\sum_{i=l+1}^{\infty} f\left(d_{i}+0\right)-f\left(d_{i}-0\right)<\epsilon
$$

and, on account of relations (4) and (5), the part of $G_{n}$ arising from the intervals $A$ differs from

$$
\sum_{i=1}^{l} g\left(d_{i}\right)\left\{f\left(d_{i}+0\right)-f\left(d_{i}-0\right)\right\}=\sum \int_{d i} g d f
$$

by not more than $\epsilon$. Hence the upper and lower limits of the part of $G_{n}$ arising from the intervals $A$ do not differ by more than $2 \epsilon$.

Let $e_{\epsilon}$ be the set of points of $(\alpha, \beta)$ at which the saltus of $g$ is not less than $\epsilon$. Then $e_{\epsilon}$ is closed, and consequently the part $e_{\epsilon}^{\prime}$ of $e_{\epsilon}$ which is not interior to $A$ is closed. Let the common part of $e_{\epsilon}$ and the set of continuities of $f$ have zero measure with respect to $f$. It then follows, if (9) is taken into consideration, that the closed set $e_{\epsilon}^{\prime}$ can be put in a finite set of nonoverlapping intervals $B=\left(b_{i}, b_{i}^{\prime}\right)$ which do not overlap the set $A$, which are such that $b_{i}, b_{i}^{\prime}$ are points of continuity of $f$, and for which

$$
\sum\left\{f\left(b_{i}^{\prime}\right)-f\left(b_{i}\right)\right\}<2 \epsilon .
$$

It then follows that for any $n$ the part of $G_{n}$ arising from the intervals $B$ is not greater in numerical value than $2 \in M$, where $M$ is the least upper bound of $|g(x)|$.

At each point of $(\alpha, \beta)$ not interior to the intervals $A+B$ the saltus of $g$ is less than $\epsilon$. Hence the closed intervals complementary to the set interior to $A+B$ can be subdivided into a finite set of intervals $C=\left(c_{i}, c_{i}^{\prime}\right)$ such that $c_{i}, c_{i}^{\prime}$ are points of continuity of $f$ and on each interval $\left(c_{i}, c_{i}^{\prime}\right)$ the fluctuation of $g$ is not more than $\epsilon$. If $G_{n}^{s}=\left\{g\left(\xi_{1}^{i}\right)+\cdots+g\left(\xi_{m}^{b}\right)\right\} / n$ is the part of $G_{n}$ arising from the interval $\left(c_{i}, c_{i}^{\prime}\right)$, then $G_{n}^{b}$ lies between the values

$$
\left\{g\left(c_{i}\right)-\epsilon\right\} p_{i} / n, \quad\left\{g\left(c_{i}\right)+\epsilon\right\} p_{i} / n,
$$


where $p_{i}$ is the number of points of the sequence $\left\{x_{k}\right\}$ on $c_{i}<x<c_{i}^{\prime}$. Hence as $n \rightarrow \infty$ the upper and lower limits of $G_{n}^{i}$ do not differ by more than $2 \epsilon\left\{f\left(c_{i}^{\prime}\right)-f\left(c_{i}\right)\right\}$. We can now conclude that the least upper bound and greatest lower bound of

$$
G_{n}=\frac{g\left(\xi_{1}\right)+\cdots+g\left(\xi_{n}\right)}{n}
$$

do not differ by more than $2 \epsilon+2 \epsilon M+2 \epsilon\{f(\beta)-f(\alpha)\}$. Since $\epsilon$ is arbitrary, it follows that if $g(x)$ satisfies the conditions of Theorem I then the CS-integral of $g$ with respect to the monotone function $f(x)$ exists.

If the set of discontinuities of $g$ has measure greater than 0 with respect to $f$ over the continuities of $f$, then for some $\epsilon>0$ there exists $d>0$ such that $\sum\left\{f\left(b_{i}^{\prime}\right)-f\left(b_{i}\right)\right\}>d$ regardless of the choice of the intervals $\left(b_{i}, b_{i}^{\prime}\right)$. Consequently for every $n$ there exist $\xi_{i}$ and $\xi_{i}^{\prime}$ such that the sums $\left\{g\left(\xi_{1}\right)+\cdots+g\left(\xi_{n}\right)\right\} / n$ and $\left\{g\left(\xi_{1}^{\prime}\right)+\cdots+g\left(\xi_{n}^{\prime}\right)\right\} / n$ will differ by an amount which, for the fixed $\epsilon$ in question, is not less than $\epsilon d$. From this it follows that the CS-integral of $f$ with respect to $g$ does not exist, and we conclude that the conditions of Theorem I are necessary. ${ }^{4}$

To obtain the LS-integral of $g$ with respect to $f$ we proceed as follows: Let $\left(y_{i-1}, y_{i}\right)$ be a subdivision of the range of $g$. Let $e_{i}$ be the part of $(\alpha, \beta)$ for which $y_{i-1} \leqq g<y_{i}$, and $f\left(e_{i}\right)$ the image of the set $e_{i}$ by means of the transformation $y=f(x)$, where a point $x_{0}$ of discontinuity of $f$ corresponds to the closed interval $\left\{f\left(x_{0}-0\right), f\left(x_{0}+0\right)\right\}$. Then $\operatorname{LS} \int_{\alpha}^{\beta} g d f=\lim \sum y_{i} m f\left(e_{i}\right)$ as $y_{i}-y_{i-1} \rightarrow 0$, provided this limit exists. This limit does exist if $g$ is measurable with respect to $f$, which readily follows if the points of continuity of $f$ at which $g$ is discontinuous have zero measure with respect to $f$. If $D=d_{1}, d_{2}, \cdots$ is the set of discontinuities of $f$, then

$$
\operatorname{LS} \int_{D} g d f=\sum g\left(d_{i}\right)\left\{f\left(d_{i}+0\right)-f\left(d_{i}-0\right)\right\}=\operatorname{CS} \int_{D} g d f .
$$

If the intervals $A=\left(a_{i}, a_{i}^{\prime}\right)$ and $B=\left(b_{i}, b_{i}^{\prime}\right)$ are properly chosen, then $\mathrm{CS} \int_{A} g d f$ is arbitrarily near to $\sum g\left(d_{i}\right)\left\{f\left(d_{i}+0\right)-f\left(d_{i}-0\right)\right\}=\operatorname{LS} \int_{D} g d f$, and both $\operatorname{CS} \int_{B} g d f$ and $\operatorname{LS} \int_{B} g d f$ are less in numerical value than $2 \epsilon M$.

${ }^{4}$ The necessity of the conditions of Theorem I would not follow if $G_{n}$ were restricted to the form $\left\{g\left(x_{1}\right)+\cdots+g\left(x_{n}\right)\right\} / n$ of Copeland's original definition. Let $f(x)=x$. The sequence $\left\{x_{k}\right\}, k=1,2, \cdots, n, n=1,2, \cdots$, is a denumerable set on $(\alpha, \beta)$. If $g(x)=0$ at each point of this denumerable set, and for the rest of $(\alpha, \beta)$ $g(x)=1$ when $x$ is rational and $g(x)=0$ when $x$ is irrational, then $\operatorname{CS} \int_{\alpha}^{\beta} g d f$ exists and is equal to zero, but $g$ does not satisfy the conditions of Theorem I. 
Over the set of intervals $\left(c_{i}, c_{i}^{\prime}\right)$ both the CS-integral and the LS-integral of $g$ with respect to $f$ lie between $\sum\left\{g\left(c_{i}\right)-\epsilon\right\}\left\{f\left(c_{i}^{\prime}\right)-f\left(c_{i}\right)\right\}$ and $\sum\left\{g\left(c_{i}\right)+\epsilon\right\}\left\{f\left(c_{i}^{\prime}\right)-f\left(c_{i}\right)\right\}$. From these considerations the truth of Theorem II readily follows.

In proceeding with the proof of Theorem III we first define the derivative of a function $F(x)$ with respect to a monotone function $f(x)$. Let

$$
\begin{aligned}
\psi(x, \Delta x) & =\frac{F(x+\Delta x)-F(x-0)}{f(x+\Delta x)-f(x-0)}, \\
& =\frac{F(x+\Delta x)-f(x-0) \neq 0, \Delta x>0,}{f(x+\Delta x)-f(x+0)}, \\
& f(x+\Delta x)-f(x+0) \neq 0, \Delta x<0, \\
& =0 \text { otherwise. }
\end{aligned}
$$

If, for a fixed $x, \psi(x, \Delta x)$ tends to a limit as $\Delta x \rightarrow 0$, then this limit is the derivative of $F(x)$ with respect to $f(x), d F / d f$. Now let $F(x)=\int_{\alpha}^{x} g d f$. If $x_{0}$ is a point of discontinuity of $f$, then, for $\Delta x>0, F\left(x_{0}+\Delta x\right)$ $-F\left(x_{0}-0\right)$ is the limit as $\delta \rightarrow 0$ of $F\left(x_{0}+\Delta x\right)-F\left(x_{0}-\delta\right)$, and this last is the limit of

$$
\frac{g\left(\xi_{\overline{1}}^{-}\right)+\cdots+g\left(\xi_{p}^{-}\right)+q g\left(x_{0}\right)+g\left(\xi_{1}^{+}\right)+\cdots+g\left(\xi_{r}^{+}\right)}{n}
$$

where $\xi_{1}^{-}, \cdots, \xi_{\bar{p}}^{-}, \xi_{1}^{+}, \cdots, \xi_{r}^{+}$are points on the intervals $\left(x_{k-1}, x_{k}\right)$ formed by the sequence $\left\{x_{k}\right\}$ on $x_{0}-\delta<x<x_{0}, x_{0}<x<x_{0}+\Delta x$ respectively, and $q$ is the number of times $x_{0}$ is repeated in this sequence. Then for $\delta$ and $\Delta x$ fixed $p / n \rightarrow f\left(x_{0}-0\right)-f\left(x_{0}-\delta+0\right), q / n \rightarrow f\left(x_{0}+0\right)$ $-f\left(x_{0}-0\right)$, and $r / n \rightarrow f\left(x_{0}+\Delta x-0\right)-f\left(x_{0}+0\right)$. Hence for $\delta$ and $\Delta x$ sufficiently small $p / n$ and $r / n$ are both arbitrarily near to zero. Since $g$ is bounded, it then follows from (10) that for $\delta$ and $\Delta x$ sufficiently small $F\left(x_{0}+\Delta x\right)-F\left(x_{0}-\delta\right)$ is arbitrarily near to $g\left(x_{0}\right)\left\{f\left(x_{0}+0\right)\right.$ $\left.-f\left(x_{0}-0\right)\right\}$, and consequently $\psi\left(x_{0}, \Delta x\right)$ is arbitrarily near to $g\left(x_{0}\right)$. Similarly it can be shown that for $\Delta x<0, \psi\left(x_{0}, \Delta x\right) \rightarrow g\left(x_{0}\right)$.

Next let $x_{0}$ be a point of continuity of both $g$ and $f$. Then $F\left(x_{0}+\Delta x\right)$ $-F\left(x_{0}-\delta\right)$ is the limit of a sum of the form

$$
\frac{g\left(x_{0}\right)+t_{1}+\cdots+g\left(x_{0}\right)+t_{p}}{n}
$$

where $\left|t_{i}\right|<\epsilon$ if $\delta$ and $\Delta x$ are sufficiently small, and $p / n \rightarrow f\left(x_{0}+\Delta x-0\right)$ 
$-f\left(x_{0}-\delta+0\right)$. Hence for $\delta$ and $\Delta x$ sufficiently small, and $n$ sufficiently great

$$
\psi\left(x_{0}, \Delta x\right)=g\left(x_{0}\right)+t\left\{f\left(x_{0}+\Delta x-0\right)-f\left(x_{0}-\delta+0\right)\right\}
$$

where $t \rightarrow 0$ as $\Delta x$ and $\delta \rightarrow 0$. We conclude therefore that, for $\Delta x>0$, $\psi\left(x_{0}, \Delta x\right) \rightarrow g\left(x_{0}\right)$. Similarly it can be shown that, for $\Delta x<0, \psi(x, \Delta x)$ $\rightarrow g\left(x_{0}\right)$. Hence, at $x_{0}, d F / d f=g\left(x_{0}\right)$, and Theorem III is established.

We note that the definition of $d F / d f$ given above implies the existence of $F(x-0)$ and $F(x+0)$, which can easily be shown if $F(x)=\operatorname{CS} \int_{\alpha}^{x} g d f$. Furthermore, at points of discontinuity of $F(x)$ or of $f(x)$ the value of the derivative does not depend on the value of these functions at the point.

The next considerations are the removal of the restrictions $f(\alpha+0)=0, f(\beta-0)=1$, and the equivalence of the original and the modified definition. As to the first of these, it can be done, following Copeland, by setting

$$
\phi(x)=\frac{f(x)-f(\alpha+0)}{f(\beta-0)-f(\alpha+0)}
$$

and defining

$$
\operatorname{CS} \int_{\alpha<x<\beta} g d f=\operatorname{CS} \int_{\alpha<x<\beta} g d \phi \cdot[f(\beta-0)-f(\alpha+0)] .
$$

It is possible, however, and perhaps advisable, to forego this restriction from the start: In (3) let $x_{k}$ be the greatest lower bound of numbers $x$ for which

$$
f(x-0) \leqq f(\alpha+0)+(k / n)\{f(\beta-0)-f(\alpha+0)\} \leqq f(x+0),
$$

and define

$$
\operatorname{CS} \int_{\alpha<x<\beta} g d f=\lim _{n \rightarrow \infty} \frac{g\left(\xi_{1}\right)+g\left(\xi_{2}\right)+\cdots+g\left(\xi_{n}\right)}{\sigma_{n}},
$$

where $\xi_{k}$ is chosen as above, and $1 / \sigma_{n}=\{f(\beta-0)-f(\alpha+0)\} / n$. If $1 / \sigma_{n}$ replaces $1 / n$ in the foregoing discussions, all the results hold without further change in the wording. When $f(\alpha+0)=0$ and $f(\beta-0)=1,(12)$ reduces to (6); and when these restrictions do not hold, it can be shown that (11) and (12) are equivalent. The proof of this we leave to the reader.

As to the equivalence of the modified form and the original form, if the integral exists under the first it does under the second. For the 
part contributed by the discontinuities of $f$ is the same; on the intervals $\left(c_{i}, c_{i}^{\prime}\right)$ both integrals lie between the values

$$
\sum g\left(c_{i}\right)\left\{f\left(c_{i}^{\prime}\right)-f\left(c_{i}\right)\right\}-\epsilon K, \quad \sum g\left(c_{i}\right)\left\{f\left(c_{i}^{\prime}\right)-f\left(c_{i}\right)\right\}+\epsilon K,
$$

where $K=f(\beta)-f(\alpha)$. These considerations easily lead to the equality of the two. That the integral can exist under the original form and not under the modified form is shown by the example in the footnote above. The set $x_{1}^{5} ; x_{2}, x_{3} ; x_{4}, x_{5}, x_{6} ; \cdots$ on which the original definition is based is such that for any $n$ the first $n$ points are not arranged in increasing order of magnitude. But obviously these can be rearranged into a non-decreasing set $\left\{x_{k}\right\}$, and on $\left(x_{k-1}, x_{k}\right)$ the $\xi_{k}$ can be chosen as above. Then

$$
\lim _{n \rightarrow \infty} \frac{g\left(\xi_{1}\right)+\cdots+g\left(\xi_{n}\right)}{n}
$$

leads to an integral which is equivalent to that arising from the modified definition given in this paper. For the results and proof of Theorem I are the same; the part of each arising from the discontinuities of $f$ is the same; furthermore, on the intervals $\left(c_{i}, c_{i}^{\prime}\right)$ both integrals lie between the bounds given by (13).

Thus, if we get away from an integral which depends only on the values of $g$ over a denumerable set, the sequence of sets given by (3) leads to the same result as the set $x_{1} ; x_{2}, x_{3} ; \cdots$ on which the original definition is based. The sequence given by (3) is more simply defined; the two are equivalent with respect to properties (4) and (5), but for that given by (3) these properties are more easily established.

In correspondence Copeland has stated that he had in mind statistical considerations when he developed the original definition. "These numbers," that is, the set $x_{1} ; x_{2}, x_{3} ; \cdots$, "can be interpreted as a sequence of measurements of some physical quantity. The expression $f(b+0)-f(a+0)$ is interpreted as the probability that a measurement $x_{k}$ will lie in the interval $a<x \leqq b$. This probability is defined as the limit of the success ratio, that is, as the average number of points lying in the interval. If a person is to receive $g(x)$ dollars when the measurement turns out to be $x$, then the expected amount which he will receive is the limit of the average of his receipts. This limit is the integral $\int_{\alpha}^{\beta} g d f$." Whatever the utilitarian background of the idea, a Stieltjes integral of the Riemann type applicable without modification to such a wide range of functions is interesting for its own sake.

The University of Saskatchewan

${ }^{5}$ This Bulletin, loc. cit., p. 582. 